\title{
Europäisches Privatrecht in
}

Vielfalt geeint

Einheitsbildung durch Gruppenbildung im Unternehmensrecht?

\section{Droit privé européen: I’unité} dans la diversité

Convergences en droit de l'entreprise?

herausgegeben von / édité par Olivier Cachard

Liliane Nau 


\section{Table de matières/Inhaltsverzeichnis}

Avant-propos

Préface

Einführung

Liste des auteurs/Autorenverzeichnis

Entreprise et droits européens

Peter Jung

Première Partie: Identité nationale et convergence des droits

La convergence des droits dans une Fédération d'Etats-Nations Jean-Denis Mouton

L'application du droit comparé par la Cour de cassation Gérard Pluyette

La CJUE et les droits nationaux: comparaisons, convergences? Francis Donnat

Deuxieme Partie: Entreprise civile, professions libérales et convergence des droits

La formation des juristes en France, perspectives d'évolution Bernard Teyssié

Les métiers du droit dans la jurisprudence de la Cour de Justicé de l'Union Européenne

Philippe Corruble

La circulation des actes notariés dans les relations

franco-allemandes

Edmond Jacoby
Pratique de la profession d'avocat: regards franco-allemands Hugues Letellier

Fragen und Perspektiven der Expertenhaftung in Europa - Die Dritthaftung von Sachverständigen für fehlerhafte Informationen Friederike Schattka

Troisième Partie: Entreprise commerciale et convergence des droits

Voyage interculturel dans le droit de l'entreprise franco-

allemand: extraits d'un carnet de voyage

Anne Guineret-Brobbel Dorsman

Première Sous-Partie: Entreprise et commercialité

Der Einzelkaufmann und seine Haftung im deutschen Recht

Michael Hakenberg

Du commerçant à l'EIRL en droit français

Evelyne Tichadou

Approche comparative du statut du conjoint du chef d'entreprise participant à l'entreprise familiale en droit français et italien Carmela Géraldine Nucera

Deuxième Sous-Partie: Distinction société civile - société commerciale

Sociétés civiles - sociétés commerciales en droit allemand:

Existe-t-il encore une différence?

Marc-Philippe Weller

Sociétés civiles, sociétés commerciales:

une distinction encore pertinente en droit français?

Christine Lebel 


\section{Troisième Sous-Partie: Entreprise et sociétés commerciale}

Sociétés de personnes et sociétés de capitaux: «summa divisio»? Stefan J. Geibel

Regards croisés sur le statut des dirigeants de sociétés Géraldine Demme/Steffen Paulmann

Anwendungsproblem bei der grenzüberschreitenden Beteiligung von Kapitalgesellschaften: deutsch-niederländisches Fallbeispiel

Markus Stephani

La concurrence entre les systèmes: modèle moniste ou modèle dualiste d'organisation des pouvoirs de direction et de contrôle de la société anonyme Katrin Deckert

Quatrième Sous-Partie: Entreprise et patrimoine

Le fonds de commerce: was ist das? Christian Franz

Haftungsbeschränkung à la carte? Vergleichende Bemerkungen zur Durchgriffshaftung im deutschen und französischen Gesellschaftsrecht

Roman Guski

La responsabilité personnelle des associés de sociétés à responsabilité limitée (SARL)

Francine Mansuy

Konvergenz im Unternehmensrecht?

Schlusswort

Peter-Christian Müller-Graff

Colloque du programme commun de recherche «convergence des droits», Nancy, 21 et 22 octobre 2010

Mike Herold

Index

\section{Entreprise et droits européens}

\section{Peter Jung}

\section{Introduction}

Le débat sur la notion d’entreprise ${ }^{1}$ et son traitement juridique représente le noyau dur du droit de lentreprise. Afin d'apprécier la portée actuelle et future de ce domaine du droit, il convient de s'attarder sur la signification scientifique et pratique de la notion d'entreprise. ${ }^{2}$

Au sens de la science économique, lentreprise est une organisation com plexe et autonome qui coordonne un ensemble de facteurs, en vue de produire pour le marché certains biens ou services. ${ }^{3}$ Les droits continentaux ont tous comme point de départ cette conception économique visant la fonction classique de l'entreprise comme cellule économique. ${ }^{4}$ Ils définissent tous l'activité de lentreprise comme étant autonome, habituelle et économique. ${ }^{5}$ Par contre, ils ont des positions différentes en ce qui concerne l'entreprise en tant qu'entité très

Selon Mestre/Tian-Pancrazi, Droit commercial - Droit interne et aspects de droit international, $28^{e}$ éd., 2009, n. 29 il s'agit d'une notion cadre. En langue française, on parle encore de «fonds de commerce» d'«établissement commercial» ou de "maison de commerce» et en langue allemande, on parle de «Gewerbe» et de «Betrieb».

2 Se référer pour le droit allemand notamment à Wieland, Handelsrecht, Erster Band Das kaufmännische Unternehmen und die Handelsgesellschaften, 1921, p. 159 et s. Oppikofer, Das Unternehmensrecht in geschichtlicher, vergleichender und rechtspolitischer Betrachtung, 1927; Raiser, Das Unternehmen als Organisation, 1969, p. 93 et s. Raisch, Unternehmensrecht, t. 1 (Unternehmensprivatrecht), 1973, p. 78 et s.; pour le droit francais notamment Despax, L'entreprise et le droit, 1957; Ripert/Roblot/Vogel, Traité de droit commercial, t. 1/1, 19e éd., 2009, n. 102; pour le droit suisse $v$. Steiger, Zur Problematik eines „Unternehmensrechts", SAG 1981, 1 et s.; Meier-Hayoz/Forstmoser Schweizerisches Gesellschaftsrecht, $10^{\circ}$ éd., 2007, $\$ 5$ n. 1 et s.; Ruedin, Droit des

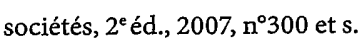

3 Se référer pour les différentes définitions économiques de l'entreprise à Oppetit/Sayag, Les structures juridiques de l'entreprise, $3^{\mathrm{e}}$ éd., 1981, n. 14 et s. ainsi que Blaise, Droit des affaires - commerçants, concurrence, distribution, $5^{e}$ éd., 2009, n. 328 et s. qui distingue entre unité de production et système social complexe.

4 Se référer p. ex. pour le droit allemand à K. Schmidt, Handelsrecht, 5 e éd., 1999, p. 66 et pour le droit français à Despax (n. 2), p. 251 et s.

5 Se référer p. ex. pour le droit allemand (notion de «Gewerbe») à Canaris, Handelsrecht, $24^{e}$ éd 2006, $\$ 2$ n 2 et s.; pour le droit français à Mercadal La notion d'entreprise, in: Les activités et les biens de l'entreprise - Mélanges offērts à Jean Derruppé, 1991, p. 9; 
variée et impliquée dans de multiples situations sur un plan interne et externe. En droit, il nexiste ainsi pas de notion unique de l'entreprise. ${ }^{6}$ En droit italien par exemple, le terme «impresa» désigne l'activité alors que le mot «azienda» désigne l'unité patrimoniale de l'entreprise.

Le droit doit d'abord prendre en considération lobjet, la nature et la dimension de lentreprise. Ainsi, les droits continentaux font assez souvent une distinction entre une entreprise commerciale, ${ }^{7}$ une petite entreprise professionnelle, ${ }^{8}$ une entreprise de profession libérale, ${ }^{9}$ une entreprise artisanale ${ }^{10}$ et une entreprise agricole ou forestière. ${ }^{11}$ Le droit ne peut pas non plus ignorer les situations différentes dans lesquelles se trouvent par exemple le petit entrepreneur à titre individuel et l'entrepreneur en forme de société faisant publiquement appel à l'épargne afin de gérer une grande entreprise. ${ }^{12}$

Vu que l'entreprise a besoin d'un encadrement juridique de son organisation et de ses relations internes ainsi que de ses relations externes, le droit de lentreprise doit prévoir des règles aussi bien sur le plan interne (droit des sociétés, droit du travail) que sur le plan externe (droit civil et commercial, droit de la concurrence).

pour le droit suisse (notion de «Gewerbe») à l’art. 2 let. b Ordonnance sur le registre du commerce (ORC).

6 Müller-Erzbach, Deutsches Handelsrecht, $2^{e}$ et $3^{\mathrm{e}}$ éd., 1928, p. 73; v. Nell-Breuning, Unternehmensverfassung, in: Biedenkopf et al. (éd.), Das Unternehmen in der Rechtsordnung - Festgabe für Heinrich Kronstein, 1967, p. 47; Blaise (n. 3), n. 342 et s.; Oppetit/ Sayag (n. 3), n. 13 et s.

7 Son exploitant étant obligé de se faire immatriculer au registre du commerce (droit allemand: $\$ \$ 1,29 \mathrm{HGB}$; droit français: art. L. 123-1 $\mathrm{n}^{\circ} 1$ et art. R. 123-32 C. com.; droit suisse: art. 934 al. 1 CO).

8 Son exploitant a la possibilité de se faire immatriculer au registre du commerce en droits allemand ( $\$ 2,105$ al. 2 HGB) et suisse (art. 934 al. 2 CO).

9 L'exploitant à titre individuel est exclu de l'immatriculation et de la commercialité en droits allemands et français (voir infra autour de l'annotation 121); se référer pour la distinction entre entreprises libérales et entreprises commerciales en droit allemand à K. Schmidt (n. 4), p. 282 et s. et en droit français à Decoopman, Entreprises libérales, entreprises commerciales, JCP 1993 I.3671.

10 Son exploitant est en principe exclu de commercialité en droit français (voir infra autour de l'annotation 123).

11 Son exploitant a la possibilité de se faire immatriculer au registre du commerce en droits allemand ( $\$ 3,105$ al. 2 HGB), autrichien ( $\$ 4$ al. 3 UGB) et suisse (art. 934 al. 2 CO).

12 Se référer pour le régime particulier des sociétés cotées en droits communautaire et français à Conac, La distinction des sociétés cotées et non cotées, Rev. soc. 2005, p. 67 et s. et pour le droit français p. ex. L. 225-37 al. 6, 225-68 al. 7 et 225-102-1 al. 5 modifiés par la loi du 26 juillet 2005; pour le droit allemand à $\$ \$ 3$ al. 2, 123 al. 3 phr. 2, 149 al. 1, 248a al. 1 AktG et Fleischer, Das Aktiengesetz von 1965 und das neue Kapitalmarktrecht, AG 2006, p. 454 et s.
Le droit doit en plus prendre en considération le fait qu'une entreprise est une entité dynamique. Selon le commercialiste bâlois Carl Wieland, il s'agit d’un corps ayant une circulation sanguine permanente. ${ }^{13}$ Son objet concret et les facteurs de production sont adaptés en permanence en fonction des besoins du marché. L'entreprise est une potentialité, elle n'a pas qu'une valeur de substance mais également une valeur de rendement. ${ }^{14}$

L'entreprise, étant à la fois un ensemble de facteurs de production et un acteur sur le marché, joue deux rôles juridiques principaux. Elle est considérée comme étant un objet de droit (A.) et comme étant un sujet de droit (B.).

\section{A. L'entreprise - objet de droit}

En droit civil et commercial de l'Union Européenne et de ses pays membres, l'entreprise est considérée comme un objet, c'est-à-dire comme étant un ensemble de facteurs de production (I.) et comme étant un moyen d'activité économique de son exploitant (II.).

\section{L'entreprise - ensemble de moyens de production}

\section{Objet composé}

L'entreprise est, en tant qu'ensemble de moyens de production, un objet composé de biens matériels et immatériels, d'un personnel technique et commercial, d'auxiliaires indépendants et d’obligations. ${ }^{15}$ Bien que cet ensemble de personnes, de droits et d'obligations constitue une unité économique et sociale, il n'est pas considéré comme étant un seul objet patrimonial. Il n'y a pas de droit de propriété quant à l'entreprise en tant que telle. Les droits continentaux ont néanmoins développé plusieurs mécanismes afin de pouvoir traiter cet ensemble affecté à un but certain comme étant entièrement ou partiellement une unité lors de transactions et comme étant un objet propre digne de protection en droit délictuel. ${ }^{16}$

13 Wieland (n. 2), p. 257.

14 Se référer pour les différentes méthodes d'évaluation d'une entreprise à Hopt, in: Baumbach/Hopt, Handelsgesetzbuch, 35e éd., 2012, Einl v $\$ 1$ n. 35 et s.

15 Se référer p. ex. à Oppikofer (n. 2), p. 87 et s.; Böhm, Der Zusammenhang zwischen Eigentum, Arbeitskraft und dem Betreiben eines Unternehmens, in: Biedenkopf et al. (éd.), Das Unternehmen in der Rechtsordnung - Festgabe für Heinrich Kronstein, 1967, p. 11, 24 et s.; Blaise (n. 3), n. 331.

16 Infra autour de l'annotation 52. 


\section{Objet dispersé}

L'entreprise est un objet souvent dispersé et qui, géographiquement, peut avoir plusieurs localisations. ${ }^{17}$ Le droit de l'Union Européenne et les droits des États membres distinguent létablissement principal (Hauptniederlassung) des succursales (Zweigniederlassungen) et des simples unités d'exploitation (unselbständige Betriebsstätten). ${ }^{18}$ Létablissement principal correspond au siège réel de lexploitant et se trouve ainsi à l'endroit où exerce effectivement la direction générale de l'entreprise. Les succursales se distinguent des simples unités d'exploitation par une certaine indépendance organisationnelle. Au contraire des filiales (Tochtergesellschaften), qui sont juridiquement indépendantes et qui gèrent leur propre entreprise, une succursale reste intégrée dans l'ensemble de l'entreprise. ${ }^{19}$

Cette dispersion territoriale de l'entreprise prend de l'importance lorsque nos droits ont besoin d'un point de rattachement réel. C'est par exemple le cas du droit international des sociétés allemand, qui applique le droit des sociétés de l'endroit où se trouve le siège réel de l'entreprise. ${ }^{20}$ C'est également le cas du droit suisse qui rattache la compétence territoriale du registre du commerce ${ }^{2}$ et des tribunaux civils au siège réel de l'entrepreneur à titre individuel et des sociétés de personnes. ${ }^{22}$ La notion détablissement (Niederlassung) joue un rôle important dans le contexte de la liberté détablissement ${ }^{23}$ dans la Convention de $V_{\text {Vienn }}{ }^{24}$ et en droit international privé. ${ }^{25} \mathrm{La}$ notion plus large détablissement stable représente un point de rattachement en droit fiscal international. ${ }^{26}$ Les

${ }_{17}$ Menjucq, Droit des affaires, $2^{e}$ éd., 2002, n. 102

18 Se référer pour le droit supranational à: Onzième directive 89/666/CEE du Conseil, du 21 décembre 1989, concernant la publicité des succursales créées dans un État membre par certaines formes de sociétés relevant du droit d'un autre État et CJCE du 22 novembre 1978 aff. 33/78, Rec. 1978, 2183 n. 12 (Somafer); pour le droit allemand à: Hopt (n. 14), \$13 n. 3 et s.; pour le droit français à: Ripert/Roblot/Vogel (n. 2), n. 256 et 258.

19 Selon Menjucq (n. 17) une succursale est en droit franç̧ais un «établissement dépendant d'un autre et crée pour le même objet».

20 Se référer pour ce rattachement traditionnel selon la théorie du siège réel (Sitztheorie) à BGH du 21 mars 1986 BGHZ 97, 269, 271 et Kindler, in: Münchener Kommentar $5^{\circ}$ éd., 2010, IntGesR, n. 455 et s.

2I Art. 934 al. 1 CO.

22 Art. 10 al. 1 let. b et art. 12 CPC; Ruedin, Droit des sociétés, $2^{2}$ éd., 2007, nº 2482 et s.

23 Selon art. 49 al. 1 phr. 2 TFUE la liberté d'établissement comporte à côté de la création libre de filiales la création libre d'agences et de succursales.

24 Art. 1 et 10 de la Convention des Nations Unies sur les contrats de vente internationale de marchandises du 11 avril 1980 (CVIM).

25 Se référer par exemple en droit international privé suisse à l’art. 21 al. 4 LDIP.

26 Se référer à titre d'exemple à la Convention fiscale avec l'Allemagne du 21 juillet 1959, JO du 9 juillet 1961 et du 8 novembre 1961; selon la définition.de l'art. $2 n^{\circ} 7$ de la Conven- conventions de l'OMC connaissent des règles d'accès au marché pour les «présences commerciales». ${ }^{27}$

\section{Objet de valeur}

Les actifs rassemblés dans une entreprise représentent dans leur ensemble et déduction faite des passifs un objet de valeur. Les droits continentaux font selon le contexte une distinction entre la valeur de substance et la valeur de rendement. La valeur substantielle des actifs de l'entreprise lors de sa liquidation (hypothétique) déduction faite de ses passifs représente la valeur minimale de l'entreprise et - en cas d'une exploitation en forme sociétaire - de la totalité des parts sociales. ${ }^{28} \mathrm{La}$ valeur de rendement est par contre prise en compte en droit allemand de la taxe professionnelle par exemple. ${ }^{29}$

En principe, l'exploitant de l'entreprise est le titulaire de tous les actifs rassemblés dans l'entreprise, mais il peut céder l'ensemble, une partie ou un poste particulier des actifs à une autre personne tout en restant, par exemple en tan que preneur à bail ou usufruitier, l'exploitant de l'ensemble des biens et ainsi de l'entreprise.

Les actifs rassemblés dans l'entreprise servent de fonds de garantie aux créanciers de son exploitant si ce dernier est également titulaire des postes actifs. Ainsi, afin de protéger les créanciers de l'ancien exploitant lors d'un transfert de gestion dans leur croyance légitime en la persistance du fonds de garantie existant au moment de la naissance de la créance, le législateur allemand a établ des règles propres au droit commercial allemand comportant assez souvent une responsabilité (solidaire) du nouvel exploitant d'un commerce pour les dettes assumées par l’ancien exploitant ( $\$ 25 \mathrm{al}$. 1 phr. $1, \$ 27$ al. 1 et $\$ 28$ al. 1 phr. 1 $\mathrm{HGB})$.

\section{Objet de transaction}

L'entreprise devient un objet de transaction si les actifs acquis, les passifs assumés et les contrats passés dans le cadre de son exploitation ou si l'exploitation

tion, la notion d'établissement stable correspond notamment à un siège de direction, une succursale, un bureau, une usine, un atelier, tout lieu d'extraction de ressources naturelles et un chantier de construction ou de montage dont la durée dépasse douze mois.

27 Se référer p. ex. à l'art. 1 al. 2 let. c AGCS; les accords d'OMC entendent par "présence commerciale» le fait d'avoir un bureau, une filiale ou une succursale.

28 Hopt (n. 14), Einl v $\$ 1$ n. 36.

29 Gewerbesteuergesetz (GewStG) in der Fassung der Bekanntmachung vom 15. Oktober 2002, BGB1. 2002 I, p. 4167. 
seule de l'entreprise sont transférés à un nouvel titulaire ou exploitant. Ces transactions se font entre vifs ou par voie de succession.

\section{a. Transfert direct (asset deal)}

L'entreprise est directement transférée si tous ses actifs et passifs sont transférés à un nouveau titulaire. Si l'entreprise est transférée entre vifs, la plupart des droits continentaux distinguent l'acte créant l'obligation de transfert (vente, donation, etc.) de lacte ou plutôt des actes réalisant le transfert des actifs et passifs de l'entreprise (actes d'aliénation), ce qui n'est pas le cas du droit italien et du droit français. Selon le droit des obligations, le contrat translatif peut concerner l'ensemble des actifs et passifs. ${ }^{30}$ Le transfert des titres (propriété, position créancière, position débitrice etc.) et des relations contractuelles s'effectue dans la plupart des pays continentaux par des actes séparés de transfert visant chaque poste des actifs et passifs et chaque relation contractuelle prise individuellement. ${ }^{31}$

Afin de faciliter ces actes de transfert, les droits prévoient plusieurs mécanismes: En droit français, une partie des éléments de l'entreprise (clientèle, marchandises, matériel et outillage, droit au bail, enseigne/nom commercial, propriétés industrielles, autorisations administratives) peut être qualifiée de fonds de commerce ${ }^{32}$ et peut ainsi être transférée selon les règles particulières relatives à la vente, à l’apport, au nantissement et à la location-gérance du fonds de commerce ainsi qu'au bail commercial qui sont aujourd'hui codifiées dans le Code de commerce. ${ }^{33}$ En droits allemand et suisse, on présume, en cas de doute, qu'un acte obligatoire visant le transfert définitif ou temporaire de l'entreprise concerne tous les biens nécessaires ou affectés à son exploitation, ceci même si le transfert concerne un immeuble. ${ }^{34}$ Ces mêmes droits'contiennent en plus des dispositions facilitant l'aliénation et la possibilité de grever des biens de l'entre-

30 Se référer pour le droit allemand à Oppikofer (n. 2), p. 132 et s. et $K$. Schmidt (n. 4), p. 140 et s.; se référer pour la vente d'un fonds de commerce en droit français à $L$. 141-1 et s. C. com.; se référer en droit suisse à Meier-Hayoz/Forstmoser (n. 2), $\$ 5$ n. 12 et s.; Ruedin, Droit des sociétés, $2^{\grave{e}}$ éd. $2007, \mathrm{n}^{\circ} 2176$ et 2189 .

31 Se référer p. ex. pour le droit allemand à Wieland (n. 2), p. 251; K. Schmidt (n. 4), p. 138 et $s$.

32 Se référer pour cette notion propre au droit français à Ripert/Roblot/Vogel (n. 2), n. 435 et s.; Cohen, Traité théorique et pratique des fonds de commerce, $2^{\mathrm{e}}$ éd., 1948 et $\mathrm{Le}$ meunier, Fonds de commerce: achat et vente, exploitation et gérance, $17^{\circledR}$ éd., 2007; Le Floch, Le Fonds de commerce: essai sur le caractère artificiel de la notion et ses limites actuelles, 1986; pour les éléments incorporels et corporels exclus du fonds de commerce Menjucq (n. 17), n. 92 et s.

33 Art. L. 141-1 et s., art. 142-1 et s., 143-1 et s., 144-1 et s., 145-1 et s. C. com.

34 Droit allemand: $\$ 311 \mathrm{c}$ avec $\$ 97,98$ BGB ('obligation d'aliéner ou de grever une chose concerne également les biens nécessaires ou affectés à son exploitation); droit suisse: prise en bloc. ${ }^{35}$ Les droits italien et autrichien connaissent la possibilité d'un transfert des actifs et passifs de l'entreprise (azienda, Unternehmen) comprenant une succession du nouvel exploitant dans les contrats conclus par l'ancien exploitant dans le cadre entrepreneurial sauf si ces contrats ont un caractère personnel. ${ }^{36} \mathrm{De}$ plus, l'entreprise peut être transférée entièrement ou selon un inventaire dans le cadre d'une restructuration si son exploitant est immatriculé au registre du commerce. ${ }^{37}$ Les règles communes à tous les droits continentaux quant au maintien des droits des salariés ${ }^{38}$ démontrent bien que les salariés sont liés à l'entreprise. ${ }^{39}$

\section{b. Transfert indirect (share deal)}

Le transfert des actifs et passifs et des relations contractuelles de l'entreprise se fait indirectement par un transfert de (presque) toutes les parts sociales de la société gérant l'entreprise. Dans de telles transactions, l'entreprise n'est pas juridiquement mais économiquement transférée. Si l'un des biens rassemblés dans l'entreprise montre un vice caché, l'acquérant n'est en principe pas autorisé à faire valoir ses droits à garantie car les parts sociales ne sont pas juridiquement concernées par ce vice. Néanmoins, la doctrine et la jurisprudence préconisent des exceptions à cette règle en cas d'acquisition de (presque) la totalité des parts sociales et d'un vice affectant l'entreprise dans son ensemble. ${ }^{40}$

art. $644 \mathrm{CC}$, qui s'applique également en droit des obligations (Basler Kommentar ZGB II/Wiegand, Art. 645 n. 25).

35 Droit allemand: $\$ 926$ BGB (aliénation), $\$ 1031$ BGB (établissement d’un usufruit) et $\$ 1120$ BGB (nantissement); droit suisse: Art. 644 CC.

36 Droit italien: Art. 2556 à $2560 \mathrm{CC}$; voir Manzini, La cessione d'azienda, Contratto e impresa 1998, 1267 et s.; droit autrichien: $\$ \$ 38$ à 40 UGB

37 En parallele aux fusions et scissions, les lois et dispositions respectives prévoient le plus souvent un transfert partiel universel des biens de l'entreprise sous des conditions propres à chaque ordre juridique; voir p. ex. en droit allemand les $\$ \$ 174$ et s. UmwG et en droit suisse les art. 69 et s. LFus; se référer dans une perspective comparatiste à Hurni, Die Vermögensübertragung im Spannungsfeld zwischen Vermögens- und Unternehmensrecht, 2008.

38 Directive 2001/23/CE du Conseil du 12 mars 2001 concernant le rapprochement des législations des États membres relatives au maintien des droits des travailleurs en cas de transfert d'entreprises, d'établissements ou de parties d'entreprises ou d'établissements, JOCE $n^{\circ} \mathrm{L} 082$ du 22 mars 2001, p. 16 et s. (codifiant la directive 77/187/CEE); pour le droit suisse art. 333 et 333a CO.

39 Blaise (n. 3), n. 335.

40 Se référer p. ex. pour le droit allemand à RG du 15 novembre 1907 RGZ 67, 86, 88 et s. ainsi que Canaris (n. 5), n. 47 et s.; pour le droit suisse Vischer, Rechts- und Sachgewährleistung bei Sacheinlage- und Übertragungsverträgen über Unternehmen, SJZ 2004 105, 109 contre TF du 10 novembre 1981 ATF 107 II 419 cons. 1; en droit comparé

Peter Jung 


\section{c. Transfert de l'exploitation}

Le transfert de l'entreprise est à distinguer du transfert de l'exploitation. Le plus souvent, le transfert de gérance est lié à un transfert direct des actifs et passifs de lentreprise au nouvel exploitant. La gérance peut être transférée dans le cadre d'un bail, d'un usufruit ou d'une fiducie sans qu'un transfert de l'entreprise soit nécessaire. Le droit allemand prévoit sous certaines conditions une responsabilité solidaire de lancien et du nouvel exploitant ainsi qu'une présomption quant au transfert des créances au nouvel exploitant s'il s'agit d'un commerce. ${ }^{41}$

\section{Objet de responsabilité civile}

En tant qu'objet, lentreprise n’est pas débitrice d’obligations mais elle représente en principe un fonds saisissable pour tous les créanciers de son exploitant. Nos droits partent du principe de l'unicité du patrimoine de lexploitant.

Cependant, le législateur français vient d'introduire par une loi du 15 juin 2010 une exception à cette règle en créant le statut de lentrepreneur individuel à responsabilité limitée qui limite sa responsabilité à un patrimoine affecté à l'entreprise. ${ }^{42}$ L'idée qui est à la base des nouvelles règles françaises est assez ancienne au vu notamment des propositions détaillées de l'autrichien Oskar Pisko de $1910 .{ }^{43}$ Le Liechtenstein a pratiqué un tel statut avec peu de succès entre 1926 et $1980 .{ }^{44}$ Le droit portugais connait déjà depuis 1986 des règles relatives à une entreprise commerciale individuelle à responsabilité limitée. ${ }^{45}$ Larticle 7 de la directive 2009/102/CE ${ }^{46}$ ouvre aux Etats membres la possibilité d'introduire une telle structure si la protection des tiers est assurée d'une manière comparable au droit européen de la SARL unipersonnelle. En Allemagne, par contre, on a rapidement enterré la proposition d'un commerçant individuel à responsabilité limitée faite par le ministère bavarois de la justice

Hauck, Mängel des Unternehmens beim Unternehmens- und Beteiligungskauf. Eine rechtsvergleichende Betrachtung des deutschen und des schweizerischen Rechts, 2008, p. 471 et s.

$41 \quad \$ \$ 25$ et s. HGB; voir Canaris (n. 5), $\$ 7$ n. 1 et s.

42 Loi n ${ }^{\circ}$ 2010-658 du 15 juin 2010, JO du 16 juin 2010, p. 10984 (Art.L. 526-6ff. C. com.).

43 Pisko, Die beschränkte Haftung des Einzelkaufmannes, GrünhutsZ 37 (1910), p. 699 et s.

44 Art. 834 à 896a PGR («Die Einzelunternehmung mit beschränkter Haftung»), abrogés par LGBl. $1980 \mathrm{n}^{\circ} 39$

45 Decreto-Lei n. $248 / 86$, de 25 de Agosto, cria e regula o estabelecimento mercantil individual de responsabilidade limitada (EIRL).

46 Directive 2009/102/CE du Parlement européen et du Conseil du 16 septembre 2009 en matière de droit des sociétés concernant les sociétés à responsabilité limitée à un seul associé, JO L 258 du 1.10.2009 (la directive a abrogé la 12e directive 89/667/CE). en $2006{ }^{47}$ Les problèmes majeurs de tous ces modèles sont la détermination et la séparation du patrimoine affecté ainsi que son adaptation aux besoins de l'entreprise au fil du temps. ${ }^{48}$

Une autre exception à la règle de l'unicité du patrimoine est la déclaration d'insaisissabilité. Au contraire des droits allemand et suisse, les droits français ${ }^{49}$ et du Liechtenstein ${ }^{50}$ connaissent une telle déclaration d'insaisissabilité pour la résidence principale. Pour que les créanciers ne souffrent pas de l'insaisissabilité des droits sur l'immeuble de la résidence principale et pour préserver le crédit de l'entreprise, on pourrait de lege ferenda imaginer la création d'une caisse de garantie à laquelle chaque entrepreneur profitant d'une telle déclaration est tenu d'y adhérer. Cette caisse de garantie aurait comme rôle le dédommagement des créanciers jusquà concurrence de la valeur des biens insaisissables..$^{51}$

\section{Objet protégé}

Finalement, l'entreprise fait encore, en tant qu'ensemble de facteurs de production, lobjet de protection contre les actes délictueux. Ce ne sont pas seulement les biens matériels et immatériels que protège le droit allemand mais aussi l'entreprise dans son ensemble. Le droit allemand ne disposant pas d'une clause générale de responsabilité délictuelle comme le droit français avec l’article 1382 $\mathrm{CC}$, le Reichsgericht a créé pour protéger une entreprise contre la concurrence déloyale le droit à l'activité professionnelle établie et effective (eingerichteter und ausgeübter Gewerbebetrieb) et l'a définie comme étant un «autre droit absolu» au sens du $\$ 823$ al. 1 BGB $^{52}$ à protéger contre des actes délictueux. ${ }^{53}$ Cette conception qui fait l'objet d'une jurisprudence constante et qui représente une particularité du droit allemand permet à l'exploitant de l'entreprise de demander l'indemnisation des dommages purement économiques causés par une atteinte directe et immédiate contre son entreprise dans son ensemble, comme

47 La proposition peut être consultée sous: http://www.ug-gesellschaft.de/wp-content/ uploads/2007/06/gesetzentwurf_kaufmann_mbh_16_05_2007.pdf.

48 Se référer entre autres à l'analyse critique de Saintourens, L'entrepreneur individuel à responsabilité limitée, Rev. soc. 2010, p. 351 et s.

49 Art. L. 526-1 à 5 C. com

50 Art. 794, 813 al. 1 PGR.

51 Se référer pour cette proposition à Champaud, L'entreprise personnelle à responsabilité limitée - Rapport du groupe d'étude chargé d'étudier la possibilité d'introduire I'E.P.R.L. dans le droit français, RTD com. 1979, p. 579, 604 et s.

52 Selon le $\$ 823$ al. $1 \mathrm{BGB}$, la vie, l'intégrité corporelle, la santé, la liberté, la propriété ou un autre droit similaire sont les biens protégés par le droit délictuel.

53 Jurisprudence constante depuis RG du 27 février 1904 RGZ 58, 24 et s. et BGH du 26 octobre 1951 BGHZ 3, 270, 279 et s. 
c'est par exemple le cas des dommages causés par un arrêt de la production dû à un boycottage ou à une grève illégale.

Les relations avec les salariés et les auxiliaires ne sont en principe pas protégées par le droit délictuel général, à moins qu'il n'y ait eu une violation intentionnelle des bonnes mœurs. ${ }^{54}$ En dehors de ces cas particuliers, la rupture des relations contractuelles de l'exploitant de l'entreprise ne donne droit à des dommages-intérêts qu’en cas de concurrence déloyale. ${ }^{55}$

\section{L'entreprise - moyen d'activités économiques de son exploitant}

En tant qu'objet, l'entreprise n'est pas seulement un ensemble de facteurs de production mais aussi un moyen d'activités économiques de son exploitant.

\section{La notion d'exploitant}

L'exploitant de l'entreprise est la personne au nom de laquelle l'entreprise est gérée. C'est le sujet de droit qui jouit des droits et qui assume les obligations émanant des actes juridiques et réels accomplis en son nom dans le cadre de l'entreprise. ${ }^{56}$ L'exploitant n'a pas l'obligation d'intervenir personnellement et activement dans son entreprise et peut même confier la gestion de la globalité de son entreprise à un tiers. Il n'est pas contraint de passer des actes commerciaux pour son propre compte et peut fort bien agir pour le compte d'autrui comme commissionnaire - un tiers peut ainsi assumer les risques de l'exploitation. Peut également devenir exploitant une personne qui n'est pas propriétaire des biens qu'elle utilise dans son entreprise comme par exemple un locataire ou un usufruitier. ${ }^{57}$ La détermination de l'exploitant peut poser problème dans le cadre d'une fiducie, d'une administration judiciaire et d'une exécution testamentaire ${ }^{5}$

L'exploitation ainsi définie peut être l'ouvre aussi bien d'une personne physique que celle d'une personne morale ou celle d'une indivision. Tandis qu'en

54 Se référer p. ex. pour le droit allemand au $\$ 826 \mathrm{BGB}$ et pour le droit suisse à l’art. 41 al. $2 \mathrm{CO}$.

55 Se référer pour le droit allemand à Köhler, in: Hefermehl/Köhler/Bornkamm, Gesetz gegen den unlauteren Wettbewerb - UWG, $30^{\circ}$ éd., $2012, \$ 4$ n. 10.103 et s. et pour le droit suisse à Spitz, in: Jung/Spitz, Bundesgesetz gegen den unlauteren Wettbewerb (UWG), 2010, \$4a UWG n. 48 et $s$.

56 Se référer pour le droit allemand p. ex. à $K$. Schmidt (n. 4), p. 88 et s.

57 Se référer p. ex. pour le droit allemand à Hopt (n. 14), $\$ 1$ n. 30 et pour le droit français à Ripert/Roblot/Vogel (n. 2), n. 470 et s.

58 Se référer p. ex. pour le droit allemand à $K$. Schmidt (n. 4), p. 92 et s; Canaris (n. 5), $\$ 9$ n. 30 et s. droit français un mineur ne peut être commerçant que sur autorisation du juge des tutelles au moment de la décision d'émancipation et du président du tribunal de grande instance s'il formule cette demande après avoir été émancipé (art. L-121-2 C. com.), un mineur peut tout simplement devenir commerçant en les droits germaniques. Cependant, la validité des actes juridiques accomplis par l'incapable ou en son nom reste soumise aux conditions générales du droit civil relatives aux actes juridiques des personnes nayant pas la capacité d'exercice. ${ }^{59}$ En plus, pour protéger le mineur contre un surendettement, le droit allemand prévoit la possibilité pour le mineur de limiter sa responsabilité pour les dettes assumées pendant sa minorité au patrimoine existant au moment de l'émancipation s'il arrête l'exercice de son entreprise dans les trois mois après son émancipation ( $\$ 1629 \mathrm{a} B \mathrm{BB}$ ).

\section{La médiatisation de l'entreprise par son exploitant}

Dans nos droits privés, l'entreprise en tant que telle n'est pas un sujet de droit malgré les propos de certains auteurs. ${ }^{60}$ L'entreprise est médiatisée par son exploitant. Tous les actes juridiques passés dans le cadre de l'entreprise sont accomplis expressément ou au moins tacitement au nom de l'exploitant qui est titulaire des droits et débiteur des obligations. Le personnel et les tiers n'ont pas de relation avec l'entreprise mais uniquement avec l'exploitant. ${ }^{61}$

L'entreprise est surtout identifiée à travers son exploitant. Celui-ci porte à ces fins un nom patronymique ou le plus souvent commercial. Pour la composition, l'utilisation et la protection du nom commercial, tous nos droits connaissent des règles spécifiques en droit commercial, en droit des marques et en droit de la concurrence déloyale. Après une libéralisation du droit à la composition du nom commercial en Allemagne en $1998^{62}$ et en Autriche en

59 En droit allemand sont applicables les $\$ \$ 104$ et s., $\$ 1643$ avec les $\$ \$ 1821$ et s. et $\$ 1645$ BGB.

60 Se référer notamment pour le droit allemand à Endemann, Das Deutsche Handelsrecht, $2^{e}$ éd., 1868, p. 84 ("selbständiges Verkehrswesen») et p. 85 ("Verkehrssubjekt») et Rai$\operatorname{ser}$ (n. 2), p. 166 et s.; pour le droit français à Despax (n. 2), p. 411 et s. («sujet de droit 'naissant'»); Didier, Esquisse de la notion d'entreprise, in: Mélanges Voirin, 1966, p. 209, 213 et s.; Champaud, Le droit des affaires, $3^{e}$ éd., 1987, p. 29

61 Se référer p. ex. pour le droit allemand à Jung, Handelsrecht, $9^{e}$ éd., 2012, chap. 5 n. 2.

62 Art. $3 \mathrm{n}^{\circ} 10$ et s. Gesetz zur Neuregelung des Kaufmanns- und Firmenrechts und zur Änderung anderer handels- und gesellschaftsrechtlicher Vorschriften (Handelsrechtsreformgesetz - HRefG) du 22 juin 1998, BGBl. 1998 I, p. 1474 et s. (en vigueur depuis le $\mathrm{l}^{\mathrm{er}}$ juillet 1998). 
$2007,{ }^{63}$ le droit suisse reste un droit relativement strict en ce domaine. ${ }^{64}$ A côté du nom patronymique de l'exploitant, l'entreprise ou une partie de celle-ci peut éventuellement être désignée par un nom détablissement. Mais ce nom a beaucoup moins d'importance que le nom commercial. C'est pourquoi il ne peut pas être immatriculé au registre du commerce en Allemagne ${ }^{65}$ et en Suisse ${ }^{66}$ tandi que l’exploitant est immatriculé sous son nom commercial dans le registre du commerce. $^{67}$

\section{Les différents types d'exploitants \\ a. L'entrepreneur à titre individuel}

L'entrepreneur à titre individuel est une personne physique qui exploite toute seule une entreprise. C'est la structure d'exploitation la plus répandue dans nos pays. Exception faite du nouveau statut français de l'entrepreneur individuel à responsabilité limitée et de l'estabelecimento mercantil individual de responsabilidade limitada portugais, ${ }^{68}$ le patrimoine affecté à la gestion de l'entreprise n'est pas séparé du patrimoine privé de lentrepreneur à titre individuel. En droits allemand et autrichien, le commerçant ou l'entrepreneur à titre individuel doit ajouter à sa dénomination commerciale (Einzelfirma) le complément «eingetragene(r) Kaufmann/Kauffrau» ou bien «eingetragene(r) Unternehmer/ Unternehmerin» ou une abbréviation compréhensible (p. ex. «e.K.» ou «e.U.»).

\section{b. Les sociétés}

\section{aa. La notion de société}

Sont sociétés dans les droits continentaux les groupements privés de personnes qui se sont engagées sur une base contractuelle à réaliser un but commun. $\mathrm{A}$ partir de cette définition, trois éléments constitutifs doivent être réunis pour qu’on puisse parler d'une société:

63 Art. $1 \mathrm{n}^{\circ} 13$ et s. Handelsrechts-Änderungsgesetz (HaRÄG) du 27 oct. 2005, BGBI $\mathrm{n}^{\circ}$ 2005/120 (en vigueur depuis $1^{\text {er }}$ janv. 2007 le Handelsgesetzbuch d'autrefois étant désormais intitulé «Bundesgesetz über besondere zivilrechtliche Vorschriften für Unternehmen (Unternehmensgesetzbuch - UGB)

64 Pour un commentaire et une analyse des art. 944 et s. CO Hilti, Firmenrecht, in: Schweizerisches Immaterialgüter- und Wettbewerbsrecht, t. III/2, $2^{2}$ éd., 2005; Ruedin

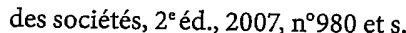

65 Rappr. $\$ 23$ phr. 1 Handelsregisterverordnung (HRV).

66 Rappr. art. 177 Ordonnance sur le registre du commerce (ORC).

$67 \$ 29 \mathrm{HGB}$ et art. $934 \mathrm{CO}$.

68 Supra autour de l'annotation 45.
La société est tout d’abord une union de plusieurs personnes, même si nos droits reconnaissent l'existence d'une société unipersonnelle émanant d'une création directe ou d'une réunion de toutes les parts sociales en une seule main Bien que le droit allemand reconnaisse depuis 1980, respectivement 1994, la création directe d'une S.A.R.L. et d'une S.A., les droits des pays romans on attendu l'élaboration et la mise en vigueur de la $12^{\mathrm{e}}$ directive de $1989 .{ }^{69} \mathrm{Le}$ droit français a introduit l'EURL en $1985^{70}$ et la SASU en $1999 .{ }^{71}$ Le droit suisse n'a accepté la fondation directe des sociétés unipersonnelles de capitaux qu'en $2008 .^{72}$

La société est ensuite une union privée basée sur un contrat de société ou des statuts. Ce deuxième élément constitutif exclut des sociétés les entités du droit public, les groupements légaux, comme par exemple la communauté par quotes-parts légale et la communauté héréditaire, et les groupements ne visant pas la création de rapports juridiques. Le contrat de société montre à la fois un caractère contractuel et institutionnel. ${ }^{73}$

Il n'y a finalement pas de société sans but commun (droits germaniques) ou sans affectio societatis (droit français). Le but commun, qui ne doit pas être confondu avec les buts personnels et souvent différents de chacun des associés, peut être lucratif ou non ainsi que temporaire ou permanent. Selon la Cour de cassation, l'affectio societatis suppose une collaboration de façon effective, dans un intérêt commun et sur un pied d'égalité. ${ }^{74}$ Alors que les droits germaniques regroupent les associations parmi les sociétés, ${ }^{75}$ le droit français s'accroche encore à la distinction entre les sociétés recherchant des bénéfices ou des économies et les associations recherchant un but non lucratif bien que cette distinction ait perdu un peu de son importance depuis la réforme de l'art. 1832

69 Douzième directive 89/667/CEE du Conseil, du 21 décembre 1989, en matière de droi des sociétés concernant les sociétés à responsabilité limitée à un seul associé (JOUE $\mathrm{n}^{\circ} \mathrm{L}$ $395 \mathrm{du} 30 / 12 / 1989$, p. 40 et s.), abrogée par la directive 2009/102/CE du Parlement européen et du Conseil du 16 septembre 2009 en matière de droit des sociétés concernant les sociétés à responsabilité limitée à un seul associé (JOUE no L 258 du 01/10/2009, p. 20 et s.).

70 Loi n ${ }^{\circ} 85-697$ du 11 juillet 1985 relative à l'entreprise unipersonnelle à responsabilité limitée et à l'exploitation agricole à responsabilité limitée, JO du 12/07/1985, p. 7082 et $s$.

71 Loi no 99-587 du 12 juillet 1999 sur l'innovation et la recherche modifiant la loi du 3 janvier 1994 instituant la société par actions simplifiée, JO du 13/07/1999, p. 10396 et s.

72 Änderung des Obligationenrechts vom 16. Dezember 2005 (GmbH-Recht sowie Anpassungen im Aktien-, Genossenschafts-, Handelsregister- und Firmenrecht), BBl. 2005, p. 7289 et s. (entrée en vigueur le $1^{\text {er }}$ janvier 2008).

73 Se référer à l'analyse comparée de Peglow, Le contrat de société en droit allemand et en droit français comparés, 2003, p. 24 et s.

74 Cass. Com. du 3 juin 1986, Rev. Soc. 1986, p. 585.

75 Windbichler, Gesellschaftsrecht, $22^{e}$ éd., $2009, \$ 2$ n. 9 et s.

Peter Jung 
C. civ. de $1978 .^{76}$ L'existence possible de la société léonine, attribuant à un associé la totalité du profit procuré par la société ou l'exonérant de la totalité des pertes ou excluant un associé totalement du profit ou mettant à sa charge la totalité des pertes, est très contestée. Tandis qu'en droit allemand la clause léonine insérée dans le contrat de société n'est réputée non écrite qu'en cas d'atteinte aux bonnes mours ( $\$ 138 \mathrm{BGB}$ ), une telle clause est toujours réputée non écrite en droits français (art. 1844-1 al. 2 CC) et italien (art. 2265 CC), la position du droit suisse restant peu claire en la matière (art. 533 al. $3 \mathrm{CO}^{77}$ ).

\section{bb. Les libertés des conventions et d'association et le numerus clausus des types de sociétés}

Les droits continentaux mettent à la disposition des futurs associés un numerus clausus des différents types de sociétés afin que ces derniers puissent en principe librement choisir la forme la plus appropriée à leur entreprise et adapter ou mélanger ces formes dans le cadre du droit dispositif. ${ }^{78}$ Grace à la liberté détablissement consacrée par les art. 49 et 54 TFUE qui permet selon la jurisprudence de la CJUE aux associés de se regrouper en principe sous une forme juridique offerte par un autre État membre de l'UE, ${ }^{79}$ ce choix est encore élargi.

Cependant, l'intérêt public exige qu'un petit nombre d'entreprises ne puisse être exploité que sous des formes juridiques déterminées. ${ }^{80}$ Autrefois, les groupements de membres de professions libérales étaient limités à la forme de société civile et ses dérivés, ${ }^{81}$ mais petit à petit, nos législateurs et nos juridictions ont accepté que ces groupements utilisent comme forme juridique la société

76 Ripert/Roblot/Germain, Traité de droit commercial t.1/2 Les sociétés commerciales, $19^{\circ}$ éd., 2009, n. 1056-23 et s.

77 Pour les différentes interprétations de l'art. 533 al. 3 CO Jung, in: Handkommentar zum Schweizer Privatrecht, 2 e éd., 2012, Art. 533 OR n. 3.

78 Pour le droit allemand Westermann, Vertragsfreiheit und Typengesetzlichkeit im Recht der Personengesellschaften, 1970; pour le droit français Brobbel-Dorsman, La GmbH \& Co.KG allemande et la «commandite à responsabilité limitée» française - Une illustration de la liberté contractuelle en droit des sociétés?, 1998, p. 49 et s.; pour le droit suisse A. Koller, Grundfragen einer Typuslehre im Gesellschaftsrecht, 1967.

79 CJCE du 9 mars 1999 aff. C-212/97, Rec. 1999 I, 1459 (Centros); CJCE du 30 septembre 2003 aff. C-167/01, Rec. 2003, I-10155 (Inspire Art).

80 Prenons l'exemple du droit allemand où la forme de société anonyme (AG) ou de société d'assurances mutuelles (VVaG) est impérative pour certaines assurances ( $\$ 7 \mathrm{VAG}$ ), oì la forme d'AG est impérative pour les caisses d'épargne-logement ( $\$ 2$ al. 1 BausparkassenG) et où la forme d'AG ou de GmbH est impérative pour les sociétés de placement de capitaux ( $\$ 6$ al. 1 phr. 2 InvG).

81 Pour le droit allemand: Ulmer, in: Münchener Kommentar zum BGB, $5^{e}$ éd., 2009, Vor $\$ 705 \mathrm{n}$. 19 et s. et 36 et s.; pour le droit français: Laurent/Vallée, Sociétés d'exercice libéral (SEL): création, gestion, évolution, $5^{e}$ éd., 2007; Lamboley, Le particularisme de capitaux..$^{82}$ Il s'avère que les législateurs allemand et français ont même créé des types de sociétés particuliers pour l'exercice d'une profession libérale. ${ }^{83}$ En droit suisse, les grands cabinets de personnes exerçant une profession libérale sont considérés au contraire du droit allemand, où les professions libérales échappent traditionnellement encore à la commercialité, ${ }^{84}$ comme étant des sociétés commerciales de personnes. ${ }^{85}$

La liberté de réunion et ses limites ne sont pas encore définies de la même manière dans les droits continentaux: Le législateur français a créé une grande gamme de sociétés différentes, dont beaucoup consiste en des dérivés des formes de société classiques destinées à un certain objet social ou à certains associés, ${ }^{86}$ alors que les législateurs allemand et suisse se contentent principalement des types de société classiques. ${ }^{87}$ Le droit suisse met à la disposition des action-

du droit des sociétés dans le cadre de l'exercice en groupe des professions libérales, in: Mélanges Michel Cabrillac, 1999, p. 597 et s.

82 Se référer p. ex. en droit allemand au $\$ 59 \mathrm{c} \mathrm{BRAO}$ (avocats), $\$ 49$ al. 1 StBer (conseillers fiscaux), $\$ 27$ al. 1 WiPrO (experts-comptables); se référer en droit français à la loi no 90 1258 du 31 décembre 1990 relative à l'exercice sous forme de sociétés des professions libérales soumises à un statut législatif ou réglementaire ou dont le titre est protégé et aux sociétés de participations financières de professions libérales qui a introduit les différentes sociétés d'exercice libéral en forme de société de capitaux (SELARL, SELAFA, SELAS, SELCA).

83 Allemagne: Partnerschaftsgesellschaft (PartG) introduite par la Gesetz über Partnerschaftsgesellschaften Angehöriger Freier Berufe (Partnerschaftsgesellschaftsgesetz PartGG) du 25 juillet 1994, BGBl. 1994 I, p. 1744 et s.; France: Loi no. 66-879 du 29 novembre 1966 relative aux sociétés civiles professionnelles.

84 Hopt (n. 14), $\$ 1$ n. 19.

85 TF du 3 juillet 1998 RTF 124 III 363.

86 Société civile (y compris société civile immobilière, société civile de moyens, société civile professionnelle, société de perception et de répartition des droits, société d'attribution d'immeubles en jouissance à temps partagé et société de construction), société en participation, société en nom collectif, société en commandite simple, société à responsabilité limitée (y compris l'entreprise unipersonnelle à responsabilité limitée, société d'exercice libéral à responsabilité limitée et l'exploitation agricole à responsabilité limitée), société anonyme (y compris société d'économie mixte, société d'économie mixte locale, société d'investissement à capital variable, société d'exercice libéral à forme anonyme), société par actions simplifiée (y compris société par actions simplifiée unipersonnelle et société d'exercice libéral par actions simplifiée), société en commandite par actions ( $\mathrm{y}$ compris société d'exercice libéral en commandite par actions) sociétés coopératives (y compris sociétés coopératives agricoles), société mutuelle et groupement d'intérêt économique.

87 Allemagne: Gesellschaft bürgerlichen Rechts ( $\$ \$ 705$ et s. BGB), offene Handelsgesellschaft ( $\$ 105$ et s. HGB), Kommanditgesellschaft ( $\$ 161$ et $s$. HGB), stille Gesellschaft ( $\$ \$ 230$ et s. HGB), Aktiengesellschaft (AktG), Kommanditgesellschaft auf Aktien 
naires un statut de société anonyme très flexible ${ }^{88}$ tandis que le droit allemand connaît un statut très rigide de la Aktiengesellschaft. ${ }^{89}$ Bien que le droit suisse ne reconnaisse pas à une société de capitaux la qualité d’associé indéfiniment responsable d'une société commerciale de personnes, ${ }^{90}$ le droit allemand accepte depuis longtemps l'amalgame entre une société de capitaux à responsabilité limitée et une société de personnes. ${ }^{91}$ L'exemple classique représente la GmbH \& Co.KG qui était autrefois très attractive sur le plan fiscal et qui est toujours très répandue en Allemagne. ${ }^{92}$ Entretemps, il existe aussi des sociétés en commandite ayant comme membre une fondation ${ }^{93}$ ou des sociétés en commandite par actions ayant comme commandité une société de capitaux. ${ }^{94}$

( $\$ \$ 278$ et s. AktG), Gesellschaft mit beschränkter Haftung (GmbHG), Unternehmergesellschaft ( $\$ 5 \mathrm{a} \mathrm{GmbHG}$ ), Genossenschaft (GenG), Versicherungsverein auf Gegenseitigkeit ( $\$ 15$ et s. VAG); eingetragener Verein ( $\$ \$ 21$ et s. BGB); Suisse: einfache Gesellschaft (art. 530 et s. CO), Kollektivgesellschaft (art. 552 et s. CO), Kommanditgesellschaft (art. 594 et s. CO), Aktiengesellschaft (art. 620 et s. CO), Kommanditaktiengesellschaft (art. 764 et s. CO), Gesellschaft mit beschränkter Haftung (art. 772 et s. CO), Genossenschaft (art. 828 et s. CO), Verein (art. 60 et s. CC).

88 Les art. 620 et s. CO sont en principe des dispositions de droit dispositif; à propos de la flexibilité du statut de la société anonyme suisse se référer à Böckli, Schweizer Aktienrecht, $4^{e}$ éd., 2009, $\$ 1$ n. 32 et s. et n. 102 et s.; Forstmoser, Gestaltungsfreiheit im Schweizerischen Gesellschaftsrecht, ZGR-Sonderheft 13, 1998, p. 254 et s.; Ruedin, Droit des sociétés, $2^{\mathrm{c}}$ éd. $2007, \mathrm{n}^{\circ} 613$ et s.

89 Selon le $\$ 23$ al. $5 \mathrm{AktG}$, les statuts d'une société anonyme allemande ne peuvent pas déroger à la loi à moins d'une autorisation expresse de celle-ci et les dispositions complémentaires sont réputées non écrites si la loi prévoit une règle exhaustive; se référer à ce propos à la critique de Mertens, Satzungs- und Organisationsautonomie im Aktien- und Konzernrecht, ZGR 1994, 426ff. («eiserne Klammer»); F. Klein, Die neueren Entwicklungen in Verfassung und Recht der Aktiengesellschaft, 1904, p. 19 («schwer Rüstung»).

90 Art. 552 al. 1 et art. 594 al. 2 CO; se référer à ce propos à la critique de Jung, Die einfache Gesellschaft als Betreiberin eines Kaufmännischen Gewerbes, in: Wessner/ Bohnet (éd.), Mélanges en l'honneur de Roland Ruedin, 2006, p. 3 et s.

91 Reconnaissance par la jurisprudence depuis RG du 4 juillet 1922 RGZ 105, 101; reconnaissance par le législateur depuis 1976 p. ex. par $\$ \$ 172$ al. 6, 177a HGB et $\$ 4$ al. 1 MitbestG 1976.

92 Binz/Sorg, Die GmbH \& Co. KG, $11^{e}$ éd., 2010, $\$ 1 \mathrm{~V}$.

93 Se référer pour ce dérivé encore contesté de la commandite simple en droit allemand à Stengel, Stiftung und Personengesellschaft - Die Beteiligung einer Stiftung an einer Personengesellschaft des Handelsrechts, 1993; en droit suisse les art. 552 al. 1 et 594 al $2 \mathrm{CO}$ empêchent une telle structure sociale.

94 Se référer pour la reconnaissance de cette structure en droit allemand au BGH du 24 février 1997 BGHZ 134, 392 et s. et Nicolin, Die Berufsfreiheit von Gesellschaften im Unternehmensrecht, 2001, p. 93 et s. cc. Les classifications des types de société

Parmi les sociétés exploitant une entreprise, nous pouvons tout d'abord distinguer les sociétés de personnes des corporations ${ }^{95}$ et des sociétés de capitaux. ${ }^{96}$ Les caractéristiques principales des sociétés de personnes sont l'existence d'un lien personnel entre les associés, l'absence de la personnalité juridique dans les droits germaniques, la nature contractuelle et personnelle de lorganisation interne (contrat de société, gestion uniquement par les associés, principe de la prise des décisions à l'unanimité, obligation de loyauté, changement d’associé limité), la responsabilité directe, personnelle et solidaire (le plus souvent mais pas forcément illimitée) des associés ainsi que la souplesse de leur statut légal. A lopposé, dans les sociétés de capitaux et les autres corporations, la personne des associés est en principe indifférente. Ces types de sociétés, qui sont strictement réglementées, démontrent ainsi une structure dorganisation collective (statuts, gestion par des tiers, en principe prise de décisions à la majorité et libre cessibilité des parts) et sont dotées de la personnalité juridique. Les associés ne répondent en principe pas personnellement des dettes de la société et ne sont par conséquent, responsables que dans la limite de leurs apports. L'opposition entre sociétés de personnes et corporations pouvant être fortement atténuée en droit privé par certaines dispositions statutaires, elle conserve toute sa valeur en matière fiscale. ${ }^{97}$

$\mathrm{Si}$ nous regardons encore un peu de plus près les questions délicates de l'autonomie et de la transparence des corporations et des sociétés de personnes, nous devons constater que les droits germaniques sont beaucoup plus réticents que les droits romans à attribuer à la société la personnalité juridique. Dans les systèmes germaniques, seules les corporations disposent de la personnalité juridique, alors que ce sont toutes les sociétés en droit français à lexception de la société de fait et la société en participation. Les sociétés de personnes du droit allemand, autrichien et suisse sont des sociétés en indivisions dans lesquelles le patrimoine social nappartient ni à la société civile en tant que telle ni aux associés en tant que personnes individuelles, mais à tous les associés en tant qu'indi-

95 Se référer pour la distinction entre Personengesellschaften et Körperschaften en droit allemand à Windbichler (n. 75), $\$ 2 \mathrm{n}$. 9 et s. et en droit suisse à Meier-Hayoz/Forstmoser (n. 2), $\$ 3 \mathrm{n} .1$ et s.

96 Se référer pour la distinction entre sociétés de personnes et sociétés de capitaux Ripert/ Roblot/Germain (n. 76), n. 1164 et s.; Ruedin, Droit des sociétés, $2^{k}$ éd. 2007, nº32 et s.

97 Se référer pour la transparence fiscale des sociétés de personnes (impôt sur le revenu) et la non-transparence des corporations (impôt sur les sociétés) en droit allemand à Blümich, EStG - KStG - GewStG, 110éd., 2011, $\$ 1$ EStG n. 100 et s.; en droit français à Cozian/Viandier/Deboissy, Droit des sociétés, 24'éd., 2011, n. 42 et s. et en droit suisse à Blumenstein/Locher, System des Schweizerischen Steuerrechts, $6^{e}$ éd., 2002, p. 53 et s. et 191 et s. 
visaires (Gesamthandsvermögen). ${ }^{98}$ Tandis que le droit suisse applique encore la théorie classique de la Gesamthand, la jurisprudence allemande a finalement attribué dans un grand arrêt en 2001 la capacité juridique à la société civile en tant que telle si la société civile se révèle au tiers (Rechts- und Parteifähigkeit der Außengesellschaft bürgerlichen Rechts). ${ }^{99}$ Il faut ainsi faire en droit allemand une distinction entre la personnalité juridique des corporations et la simple capacité juridique des sociétés civiles se révélant au tiers. ${ }^{100}$ Cette distinction sert encore notamment à justifier et à maintenir les principes de l'autonomie des corporations et de la transparence des sociétés de personnes, bien que ces principes connaissent beaucoup d'exceptions comme notamment la levée du voile corporatif afin de rechercher la responsabilité des associés puissants ou de mieux appliquer certaines normes demandant une qualité humaine ou d'identifier l'associé unique ou dominant de la société. ${ }^{101} D^{3} u n$ autre côté, la société de personnes est de plus en plus traitée comme une entité propre par rapport à ces associés et notamment par rapport aux commanditaires. ${ }^{102}$

Dans le contexte de la distinction des sociétés de personnes des corporations, il convient encore de mentionner une particularité du droit allemand qui est le grand nombre des sociétés de personnes faisant publiquement appel à l'épargne, notamment dans la structure d'une GmbH \& Co.KG ou d'une société en participation. Vu que le droit allemand des sociétés de personnes n’est pas adapté à une structure réelle ayant un grand nombre de commanditaires comme associés investisseurs, le contrat de société prévoit le plus souvent l'institution d'une assemblée des commanditaires et un comité des commanditaires qui fonctionnent le plus souvent comme l'assemblée générale et le conseil de surveillance d'une société anonyme. Pour assurer la protection des associés investisseurs et le fonctionnement de la société, la jurisprudence a développé un

98 Se référer pour le droit allemand au $\$ \$ 718$ et $s . B G B$ et p. ex. Ulmer, Die Gesamthandgesellschaft - ein noch immer unbekanntes Wesen? AcP 198 (1998), p. 113 et s.; pour le droit autrichien au $\$ 1182 \mathrm{ABGB}$ et p. ex. Krejci, in Krejci (éd.), Reform-Kommentar UGB/AGB, $2007, \$ 105 \mathrm{n}$. 28 et s. et pour le droit suisse à l'art. 540 al. 1 et $2 \mathrm{CO}$ et p. ex. Meier-Hayoz/Forstmoser (n. 2), $\$ 2$ n. 62 et s.

99 BGH du 29 janvier $2001 \mathrm{BGHZ} 146,341$

100 Ulmer, Die höchstrichterlich „enträtselte“ Gesellschaft bürgerlichen Rechts, ZIP 2001, 585,587 et s.

101 Se référer pour le droit allemand à Jung, Der Unternehmergesellschafter als personaler Kern der rechtsfähigen Gesellschaft, 2002 (Durchgriff); pour le droit français à Cozian/ Viandier/Deboissy (n. 97), n. 172 et s. (abus de la personnalité juridique) et Paerels, Le dépassement de la personne morale, 2009; pour le droit suisse à Hovagemyan, Transparence et réalité économique des sociétés, 1994; Ruedin, Droit des sociétés, $2^{e}$ éd., 2007, $\mathrm{n}^{\circ} 745$.

102 Se référer pour le droit allemand au $\$ 124 \mathrm{HGB}$ (OHG, KG) et BGH du 29 janvier 2001 BGHZ 146, 341 (Außen-GbR); pour le droit français à l'art. 1842 al. I CC; pour le droit suisse à l'art. $562 \mathrm{CO}$ (Kollektivgesellschaft) et à l'art. $602 \mathrm{CO}$ (Kommanditgesellschaft).

régime particulier pour ces types de sociétés soumettant leur contrat de société à une interprétation plutôt objective ${ }^{103}$ et en cas de doute, à une interprétation en faveur des intérêts des associés investisseurs ainsi quà un contrôle jurisprudentiel selon le principe de la bonne foi. ${ }^{104} \mathrm{La}$ prise de décisions à la majorité peut être prévue d'une manière générale et sans précisions quant à son objet par le contrat de société. ${ }^{105}$

D'autres classifications importantes se font selon le critère de la commercialité de l'activité ou de l'immatriculation au registre du commerce (sociétés civiles et sociétés commerciales), voir selon l'ostensibilité sur le plan extérieur (sociétés se révélant aux tiers et sociétés occultes) ou selon le mode de financement (sociétés faisant ou non appel à l'épargne/étant cotée ou non à un marché réglementé). ${ }^{106}$

\section{c. Autres exploitants}

A côté de l’entrepreneur à titre individuel et des sociétés, une entreprise peut être exploitée par un groupement de personnes tel que la communauté héréditaire ${ }^{107}$ et l'indivision des époux formée exceptionnellement dans le cadre de la communauté des biens. ${ }^{108}$ L'entreprise peut également être exploitée par une fondation, c.à.d. de manière impersonnelle. Cette forme d'exploitation est de plus en plus acceptée dans nos droits même si la fondation n’a pas d’autre but que la gestion et la persistance de lentreprise. ${ }^{109}$ Mais la fondation, comprenant

103 BGH du 30 avril 1979 NJW 1979, 2102

104 BGH du 14 avril 1975 BGHZ 64, 238.

105 BGH du 13. mars 1978 BGHZ 71, 53 .

106 Windbichler (n. 75), \$2; Richter, Der Kapitalmarkt und sein Gesellschaftsrecht - Überlegungen zu einem kapitalmarktgemäßen Gesellschaftsrecht börsennotierter Gesellschaften, ZHR 172 (2008), 419 ff.; Ripert/Roblot/Germain (n. 76), n. 1158 et s.; MeierHayoz/Forstmoser (n. 2), $\$ \$ 3,4 . ;$ Ruedin, Droit des sociétés, $2^{2}$ éd. 2007, $n^{\circ} 822$

107 Canaris (n. 5), $\$ 9$ n. 1 et s.

108 BayObLG du 25 juillet 1991 NJW-RR 1992, 33; voir la critique de K. Schmidt (n. 4), p. 107 et s.

109 En Allemagne, une fondation peut gérer directement (Unternehmensträgerstiftung) ou indirectement (Beteiligungsträgerstiftung) une entreprise sauf si elle n'a pas d'autre but que la gestion et la persistance de l'entreprise (Unternehmensselbstzweckstiftung) voir Reuter, in: MünchKomm BGB $\$ \$ 80,81$ BGB n. 88 et s.; Berndt, Stiftung und Unternehmen, $7^{\mathrm{e}}$ é., 2003; Kronke, Stiftungstypus und Unternehmensträgerstiftung, 1988, p. 195 et s.; en France: Loi n ${ }^{\circ}$ 90-559 du 4 juillet créant les fondations d'entreprise et modifiant les dispositions de la loi $n^{\circ} 87-571$ du 23 juillet 1987 sur le développement du mécénat relatives aux fondations, JORF $\mathrm{n}^{\circ} 155$ du 6 juillet 1990, p. 7914 et s.; en Suisse: TF du 18 mai 2001 ATF 127 III 337 cons. 2 et Grüninger, in: Basler Kommentar, $4^{e}$ éd., 2010, Art. 80 ZGB n. 17 et s. contre Riemer, Rechtsprobleme der Unternehmensstiftung, ZBJV 1980, 489, 498 et s. 
une affectation stable et durable de son patrimoine et la fixation par le fondateur de ses buts et de son organisation, ne permet pas une gestion très judicieuse d'une entreprise dynamique. ${ }^{110}$

\section{Le statut personnel de l'exploitant}

Le statut de l'exploitant est déterminé surtout en fonction de la nature et de la dimension de son entreprise bien que l'activité de nature commerciale n'est pas la seule voie pour devenir commerçant dans les droits continentaux. En effet, la conception germanique prévoit qu'il suffit de se faire immatriculer dans le registre du commerce pour être soumis aux règles particulières du droit commercial nonobstant la nature et la dimension de lactivité professionnelle. Cela vaut surtout pour les sociétés commerciales. Nous trouvons dans nos droits peu importe s'il s'agit d'un système à code unique (droits suisse, italien et néerlandais) ou non (autres droits continentaux) - trois statuts différents: le statut d'entrepreneur ou de professionnel, le statut de commerçant et le statut de consommateur.

\section{a. Le statut d'entrepreneur}

Le statut d'entrepreneur ou de professionnel est attribué lorsqu'une personne exerce une activité visible, habituelle et indépendante. ${ }^{111} \mathrm{Il}$ entraine l'application du droit de la consommation ${ }^{112}$ si un tel exploitant agit dans le cadre de son entreprise et s'il se retrouve en face d'un consommateur. Lart. 2 let. b de la directive relative aux pratiques commerciales déloyales ${ }^{113}$ définit ainsi le pro-

110 Se référer p. ex. à Kronke (n. 109), p. 228 et s.; Riemer, Rechtsprobleme der Unternehmensstiftung, ZBJV 1980, 489, 509 et s.

111 Se référer p. ex. pour une définition de la notion d'entreprise en droit suisse à l'art. 2 let. b Ordonnance sur le registre du commerce (ORC): «entreprise: une activité économique indépendante exercée en vue d'un revenu régulier».

112 Se référer pour une codification de l'acquis communautaire en la matière à la directive 2011/83/UE du Parlement européen et du Conseil du 25 octobre 2011 relative aux droits des consommateurs, modifiant la directive 93/13/CEE du Conseil et la directive 1999/44/CE du Parlement européen et du Conseil et abrogeant la directive 85/577/CEE du Conseil et la directive 97/7/CE du Parlement européen et du Conseil, JOUE $n^{\circ} \mathrm{L} 304$ du 22/11/2011, p. 64 et s.

113 Directive 2005/29/CE du Parlement européen et du Conseil du 11 mai 2005 relative aux pratiques commerciales déloyales des entreprises vis-à-vis des consommateurs dans le marché intérieur et modifiant la directive $84 / 450 / \mathrm{CEE}$ du Conseil et les directives 97/7/CE, 98/27/CE et 2002/65/CE du Parlement européen et du Conseil et le règlement (CE) $n^{\circ}$ 2006/2004 du Parlement européen et du Conseil («directive sur les pratiques commerciales déloyales»). fessionnel comme «toute personne physique ou morale qui, pour les pratiques commerciales relevant de la présente directive, agit à des fins qui entrent dans le cadre de son activité, commerciale, industrielle, artisanale ou libérale, et toute personne agissant au nom ou pour le compte d'un professionnel». L'art. $2 \mathrm{n}^{\circ} 2 \mathrm{de}$ la directive relative aux droits des consommateurs va dans le même sens. ${ }^{114}$ L'activité peut ainsi être commerciale, agricole, forestière, artisanale, industrielle ou de profession libérale. Les termes «professionnel» ou «activité professionnelle indépendante» sont de plus en plus employés au lieu du terme «entrepreneur» à cause d'une certaine réticence à ranger les membres des professions libérales, les artisans et les exploitants agricoles et forestiers parmi les entrepreneurs. ${ }^{115}$

\section{b. Le statut de commerçant}

La question de savoir sous quelles conditions un entrepreneur peut être considéré comme étant un commerçant est une question qui intéresse surtout les ordres juridiques ayant un droit commercial formellement séparé faisant lobjet d'un propre code comme c'est le cas des droits français, belge, autrichien, espagnol et allemand par exemple. ${ }^{116}$ Malgré l'existence de beaucoup de normes propres au commerce dans le code unique suisse, le droit suisse n’a pas développé des règles générales pour tracer une ligne entre le droit commercial et le droit privé général, comme l'ont fait notamment les Français, les Belges et les Allemands. Tandis que les droits français ${ }^{117}$ et belge ${ }^{118}$ prennent en considération les critères relatifs aux actes de commerce habituellement passés par la

114 L'art. $2 \mathrm{n}^{\circ} 2$ de la directive 2011/83/UE (n. 112) définit le «professionnel» comme «toute personne physique ou morale, qu'elle soit publique ou privée, qui agit, y compris par l'intermédiaire d'une autre personne agissant en son nom ou pour son compte, aux fins qui entrent dans le cadre de son activité commerciale, industrielle, artisanale ou libérale en ce qui concerne des contrats relevant de la présente directive».

115 Se référer p. ex. au $\$ 14$ al. 1 BGB: «Unternehmer ist eine natürliche oder juristische Person oder eine rechtsfähige Personengesellschaft, die bei Abschluss eines Rechtsgeschäfts in Ausübung ihrer gewerblichen oder selbständigen beruflichen Tätigkeit handelt.» (Est entrepreneur toute personne physique ou morale, ou société de personne ayant la capacité juridique, qui agit dans l'exercice de son activité professionnelle commerciale ou indépendante lors de la conclusion d'un acte juridique.).

116 Se référer notamment à Raisch, Die Abgrenzung des Handelsrechts vom Bürgerlichen Recht als Kodifikationsproblem im 19. Jahrhundert, 1962 et Escarra, Cours de droit commercial, nouv. éd. 1952, n. 38 et s.

117 Selon l'art. L. 121-1 C. com. sont commerçants ceux qui exercent des actes de commerce (art. L. 110-1 et s.) et en font leur profession habituelle.

118 Selon l'art. 1 C. com. belge sont commerçants ceux qui exercent des actes qualifiés de commerciaux par la loi et qui en font leur profession habituelle, soit à titre principal, soit à titre d'appoint. Selon l'art. 2 et s. C. com. belge, la loi répute certaines transactions actes de commerce. 
personne en question (systèmes principalement objectifs), les droits allemand ${ }^{119}$ et autrichien ${ }^{120}$ ont recours aux critères relatifs à la personne en question qui doit être immatriculée au registre du commerce ou avoir une activité qui exige selon sa nature et sa dimension une organisation commerciale (systèmes principalement subjectifs).

En général le statut d'entrepreneur est à la base de la commercialité bien que les droits continentaux montrent pas mal de différences en la matière: Tandis que les membres des professions libérales exploitant leur entreprise à titre individuel sont exclus de l'immatriculation au registre du commerce (et des sociétés) et ainsi de la commercialité selon les droits jurisprudentiels et coutumiers allemand et français, ils peuvent depuis 2007 se faire immatriculer au registre du commerce (Firmenbuch) afin de se soumettre librement à beaucoup de règles du droit commercial classique en Autriche. ${ }^{121}$ En droits allemand et français, les membres des professions libérales doivent créer une société de capitaux pour arriver indirectement au même résultat. ${ }^{122}$ Tandis que le droit français ne regroupe pas les artisans traditionnels parmi les commerçants, ${ }^{123}$ le droit allemand applique les règles particulières du droit commercial aux artisans sous les conditions générales qui sont soit la nécessité présumée d'une organisation commerciale de lexploitant soit son immatriculation au registre du commerce. ${ }^{124}$ Le droit suisse a longtemps hésité à soumettre les membres des professions libérales, les artisans et les exploitants agricoles et forestiers aux règles commerciales. Cependant, la jurisprudence applique de plus en plus des critères identiques à toutes les branches d'activité en appréciant notamment la

$119 \$ 1$ HGB: «(1) Kaufmann im Sinne dieses Gesetzbuchs ist, wer ein Handelsgewerbe betreibt.» (Commerçant au sens de ce code est une personne qui gère un commerce.). «(2) Handelsgewerbe ist jeder Gewerbebetrieb, es sei denn, dass das Unternehmen nach Art oder Umfang einen in kaufmännischer Weise eingerichteten Geschäftsbetrieb nicht erfordert.» (Commerce est toute entreprise professionnelle à moins que l'entreprise n'ait pas besoin d'une organisation commerciale en raison de sa nature ou de sa dimension.).

$120 \$ 1$ al. 1 et 2 Unternehmensgesetzbuch (UGB): «(1) Unternehmer ist, wer ein Unternehmen betreibt.» (Est entrepreneur toute personne qui gère une entreprise.) «(2) Ein Unternehmen ist jede auf Dauer angelegte Organisation selbständiger wirtschaftlicher Tätigkeit, mag sie auch nicht auf Gewinn gerichtet sein.» (Une entreprise est une organisation créée à long terme et destinée à poursuivre une activité économique indépendante peu importe que son but soit lucratif ou non.). Voir également les $\$ \$ 8$ et 189 UGB.

$121 \$ 4$ al. 2 et $\$ 5$ UGB.

122 Jung (n. 61), chap. 2 n. 11 (droit allemand) et s.; Blaise (n. 3), n. 211 (droit français).

123 Jurisprudence constante depuis Cass. civ. du 22 avril 1909 DP.1909.I.344; Blaise (n. 3), n. 199 et s.

$124 \$ 1$ al. 2 et $\$ 2 \mathrm{HGB}$. nature et la dimension de l'entreprise nécessitant une organisation commerciale. $^{125}$

Les commerçants sont soumis aux règles relatives à leur statut (immatriculation au registre du commerce, nom commercial, comptabilité, représentation). S'ils agissent pour les besoins de leur commerce, les règles relatives aux actes de commerce mixtes ${ }^{126}$ et - s'ils se trouvent en face d'un homologue agissant également pour les besoins de son commerce - les règles relatives aux actes de commerce bilatéraux s'appliquent. ${ }^{127}$

\section{c. Le statut de consommateur}

Le consommateur est l'opposé de lentrepreneur en droit de la consommation. ${ }^{128}$ Son statut n'est pas attribué de manière générale mais en fonction d’une situation particulière: Le consommateur passe un acte juridique à des fins non professionnelles. A l'inverse de l'entrepreneur, il est toujours une personne physique. Par exemple, selon lart. $2 \mathrm{n}^{\circ} 1$ de la directive relative aux droits des consommateurs, un consommateur est «toute personne physique qui, dans les contrats relevant de la présente directive, agit à des fins qui nentrent pas dans le cadre de son activité commerciale, industrielle, artisanale ou libérale». ${ }^{129}$ C'est pourquoi un entrepreneur ou commerçant peut être qualifié de consommateur s'il n'agit pas pour les besoins de son entreprise ou de son commerce.

125 Pour les professions libérales TF du 3 juillet 1998 RTF 124 III 363 (cabinet d'avocat), TF du 9 septembre 2004 RTF 130 III 707 (architecte) et TF du 21 janvier 2009-4A_526/2008 (dentiste); pour les exploitants agricoles et forestiers TF du 13 mars 2009 RTF 135 III 304 (établissement horticole).

126 Se référer p. ex. pour le droit commercial allemand au $\$ 345$ avec $\$ \$ 347-350,352$ al. 2 , $354,355-368,373-376,380$ HGB

127 Se référer p. ex. pour le droit commercial allemand au $\$ \$ 346,352$ al. $1,353,354 \mathrm{a}, 369-$ $372,377,379$ HGB.

128 Se référer p. ex. pour le droit allemand au $\$ 13$ BGB: «Verbraucher ist jede natürliche Person, die ein Rechtsgeschäft zu einem Zwecke abschließt, der weder ihrer gewerblichen noch ihrer selbständigen beruflichen Tätigkeit zugerechnet werden kann.» (Est consommateur toute personne physique qui conclut un acte juridique à des fins qui n'entrent ni dans son activité professionnelle commerciale, ni dans son activité indépendante.) et $\$ 14$ al. 1 BGB: «Unternehmer ist eine natürliche oder juristische Person oder eine rechtsfähige Personengesellschaft, die bei Abschluss eines Rechtsgeschäfts in Ausübung ihrer gewerblichen oder selbständigen beruflichen Tätigkeit handelt.» (Est entrepreneur toute personne physique ou morale, ou société de personne ayant la capacité juridique, qui agit dans l'exercice de son activité professionnelle commerciale ou indépendante lors de la conclusion d'un acte juridique.).

129 Art. $2 \mathrm{n}^{\circ} 1$ de la directive 2011/83/UE (n. 112). 


\section{La protection de l'activité de l'exploitant}

L'activité de l'exploitant sur le marché n'est protégée que par les règles du droit de la concurrence. Dans une économie de marché, les relations avec la clientèle ne sont en principe pas protégées par le droit délictuel sauf si lon peut constater une violation des bonnes mœurs ${ }^{130}$ ou l'existence d'une concurrence déloyale. ${ }^{131}$ En droit français, la protection de la clientèle du commerce pourrait éventuellement faire lobjet de la protection du fonds de commerce. ${ }^{132}$

\section{B. L'entreprise - sujet de droit}

Avant d'aborder dans une deuxième partie le rôle mineur ou même inexistant de l'entreprise en tant que sujet de droit, il convient de signaler que le libellé de beaucoup de normes fait allusion à l'entreprise en tant que telle bien que la norme ne veuille réellement s'adresser quà son exploitant. Dans ces cas, lexpression n'est qu'une commodité ou un raccourci de langage. ${ }^{133}$ Ainsi, le législateur autrichien, par exemple, a intitulé la loi de réforme du droit commercial de 2006 «Bundesgesetz über besondere zivilrechtliche Vorschriften für Unternehmen», ce qui est doublement trompeur, car la loi concerne en premier lieu les exploitants en tant que sujets de droit et comprend pas mal de normes de droit public. La désignation «Einzelfirma» qui peut être traduite par «entreprise en raison individuelle» et qui était une désignation suisse tout à fait fausse de l'entrepreneur à titre individuel a été récemment remplacée par une nouvelle notion un peu moins fausse - qui est «l'entreprise à titre individuel». Le législateur français, lui, voulait éviter le mot «société» dans la désignation de la SARL unipersonnelle et l'a, par conséquent, appelée «Entreprise unipersonnelle», ce qui peut être excusable.

A l'inverse de ces dénominations trompeuses, qui se trouvent parfois également dans la doctrine, ${ }^{134}$ l'attribution de la personnalité juridique à lentreprise aurait comme conséquence le rattachement direct des actes juridiques à l'entreprise elle-même ${ }^{135}$ et non à son exploitant. Ce dernier reste alors, dans une telle approche, seulement une personne physique ou morale parmi d'autres

130 Se référer p. ex. à l’art. 41 al. 2 CO (droit suisse) et au $\$ 138$ BGB (droit allemand).

${ }^{131}$ Se référer p. ex. notamment à l’art. 4 let. a et let. $\mathrm{d} L C D$ (droit suisse) et au $\$ 4 \mathrm{n}^{\circ} 10$ UWC (droit allemand).

132 Selon Ripert/Roblot/Vogel (n. 2), n. 441, l'exploitant du fonds de commerce dispose de moyens de maintenir sa clientèle, bien qu'il n'ait pas le droit exclusif de l'exploiter.

${ }_{133}$ Blaise (n. 3), n. 345 et 349; Blanc, Les frontières de l'entreprise en droit commercial, D.1999.chron.415 et s.

134 Voir à titre d'exemple Marino, Plaidoyer pour la liberté d'expression, droit fondamental de l'entreprise, RTD com. 2011, 1 et s.

${ }^{135}$ K. Schmidt (n. 4), p. 78 et s. personnes (associés, travailleurs, créanciers etc.) regroupées au sein de l’entreprise. ${ }^{136}$ Dans les droits européens, cette conception na pas été retenue par le législateur et la jurisprudence ${ }^{137}$ et cela indépendamment des conditions d'attribution de la personnalité juridique. ${ }^{138}$ Néanmoins, en droit des sociétés et en droit collectif du travail, quelques auteurs ont souligné la nature d'entité sociale de lentreprise (I.). En droit public et pénal, des règles se réfèrent sur le plan des conditions d'application parfois à lentreprise dans sa qualité d'entité économique (II.).

\section{L'entreprise - entité sociale propre}

La perception de l'entreprise comme entité sociale a largement influencé les débats juridiques et politiques en Allemagne dans les années 1920 et 1970. En 1918, Walther Rathenau a publié une monographie concernant la nature de la société anonyme. ${ }^{139}$ Rathenau, qui a succédé à son père dans la direction de l'Allgemeine Elektricitäts-Gesellschaft (AEG) en 1915, présentait l'idée d'un intérêt propre de l'entreprise, défini comme étant l'intérêt de l'entreprise à sa propre persistance et à une promotion rentable, harmonieuse et flexible du but de l'entreprise. Cette monographie visait parmi d'autres ${ }^{140}$ à critiquer l'influence des propriétaires d'actions et soulignait ainsi l'important rôle social et économique des grandes entreprises.

Les idées de Rathenau ont été reprises dans les années 1970, quand le débat en Allemagne autour de la cogestion des salariés se trouvait au zénith. Herbert Köhler désignait l'entreprise comme étant une entité créatrice de plus-value et

136 A. Lyon-Caen/G. Lyon-Caen, La doctrine de l'entreprise, in: Dix ans de droit de l'entreprise, 1978, p. 604.

137 Droit allemand K. Schmidt (n. 4), p. 78 et s.; droit français: Blaise (n. 3), n. 346 et s.; droit suisse: Druey, Gesellschafts- und Handelsrecht, 10 éd., 2010, n. 53 et 56.

138 Tandis qu'en droits allemand et suisse l'attribution de la personnalité juridique nécessite une immatriculation ou même une concession, la Cour de Cassation (Cass. civ. du 28 janvier 1954 D.1954.217) s'est montrée particulièrement ouverte en demandant seulement un intérêt collectif et une organisation pour la formation de la volonté commune: «Attendu que la personnalité civile n'est pas une création de la loi; qu’elle appartient, en principe, à tout groupement pourvu d'une possibilité d'expression collective pour la défense d'intérêts licites ...».

139 Rathenau, Vom Aktienwesen - Eine geschäftliche Betrachtung, 1918, p. 23, 27 et 41 ets.

140 Voir aussi Haussmann, Vom Aktienwesen und vom Aktienrecht, 1928, p. 27 et s. et Geiler, Moderne Rechtswandlungen auf dem Gebiete des Privatrechts, in: Klausing et al. (éd.), Beiträge zum Wirtschaftsrecht - Festschrift für Ernst Heymann, t. I, 1931, p. 173,182 et s. 
de prestations économiques orientée par un pluralisme d'intérêts. ${ }^{141}$ L'entreprise nétait plus considérée comme une entité de biens mais comme une entité sociale dans laquelle des gens collaborent. Thomas Raiser a tiré de ces réflexions la conséquence d'une attribution de la personnalité juridique à l'entreprise ellemême. ${ }^{142}$

Bien que ces idées soient restées minoritaires, elles ont laissé des traces en droit allemand. ${ }^{143}$ Selon le $\$ 70$ al. 1 AktG 1937, le directoire était obligé de diriger la société en tenant compte du salut de l'entreprise et son personnel ainsi que de l'intérêt commun («wie das Wohl des Betriebes und seiner Gefolgschaft und der gemeinsame Nutzen von Volk und Reich es erfordern»). Bien que cette disposition n’ait pas été reprise dans la loi actuelle du 6 septembre $1965,{ }^{144}$ la plupart des auteurs considère que les membres du directoire qui sont selon le $\$ 76$ al. 1 AktG 1965 tenus de diriger la société sous leur propre responsabilité doivent non seulement prendre en considération les intérêts des actionnaires, mais doivent également tenir compte des intérêts des salariés et de l'intérêt général. ${ }^{145}$

Bien que la perception de l'entreprise en tant que cellule sociale est étroitement liée en Allemagne au débat relatif à la cogestion des salariés au niveau du directoire et notamment du conseil de surveillance d'une société de capitaux. ${ }^{146}$

141 H. Köhler, „Betrieb “ und „Unternehmen" in wirtschaftsverfassungsrechtlicher Sicht, JZ 1953, $713 \mathrm{ff}$.

142 Raiser (n. 2), p. 166 et s.

143 Se référer pour une étude historique de l'idée de l'autonomie de l'entreprise et de son influence en droit allemand à Riechers, Das „Unternehmen an sich“ - Die Entwicklung eines Begriffs in der Aktienrechtsdiskussion des 20. Jahrhunderts, 1996; la notion «Unternehmen an sich» a été créé par Haussmann dans le titre d'un article traitant les théories de Rathenau et publié dans la JW 1927, 2953 et s.

144 Schmidt-Leithoff, Die Verantwortung der Unternehmensleitung, 1989, p. 31 et s.

145 A. Hueck, Das neue Aktiengesetz und das Arbeitsrecht, RdA 1965, 321, 325; v. Falkenhausen, Verfassungsrechtliche Grenzen der Mehrheitsherrschaft nach dem Recht der Kapitalgesellschaften (AG und GmbH), 1968, p. 68; sur une base constitutionnelle (Art. 14 al. 2 GG) également Schmidt-Leithoff(n. 144), p. 214 et s.

146 Se référer pour une cogestion parfaitement paritaire à la loi du 21 mai 1951 (MontanMitbestG, BGBl. 1951 I, p. 347 et s.) et à la loi du 7 août 1956 (MitbestErgG, BGBl. $1956 \mathrm{I}$, p. 707) qui s’appliquent aux sociétés anonymes et aux sociétés à responsabilité limitée qui ont plus de 1000 salariés et qui gèrent ou dominent une entreprise minière ou sidérurgique; se référer pour une cogestion presque paritaire à la loi du 4 mai 1976 (MitbestG, BGBl. $1976 \mathrm{I}$, p. 1153 et s.) qui s’applique en principe à toutes les sociétés de capitaux et aux sociétés coopératives en dehors de la sidérurgie qui ont plus de 2000 salariés; se référer pour le régime de la cogestion dite simple à la loi du 18 mai 2004 (DrittelbG, BGBl. 2004 I, p. 974 et s.) qui prévoit une participation pour un tiers des salariés au sein du conseil de surveillance d'une société qui n'est pas une société de personnes et qui emploie plus de 500 et moins de 2000 salariés.
Cette manière de voir fait ainsi partie du système capitaliste dit rhénan. ${ }^{147}$ Nous trouvons des idées semblables dans d’autres droits continentaux comme en droit français ${ }^{148}$ et en dröit suisse. ${ }^{149}$

\section{L'entreprise - entité économique propre}

En droit public économique et en droit pénal, l'entreprise est traitée comme étant une entité économique. Dans ces domaines, il faut faire une distinction entre l'appréciation des éléments constitutifs de l'application des conséquences juridiques. Pour la détermination des éléments constitutifs, l'entreprise en tant qu'entité économique est prise en considération, alors que le ou les exploitant(s) de cette entité économique supporte(nt) les conséquences juridiques. ${ }^{150}$

\section{L'entreprise en droit public économique}

En droit public économique, l'entreprise agissant comme entité sur un marché est soumise aux règles propres concernant la surveillance des acteurs de ce marché. Cela vaut notamment pour les règles visant les pratiques anticoncurrentielles au niveau européen et national. Ainsi, la CJUE a défini l’entreprise pour les besoins des art. 101 et $\mathrm{s}$. du TFUE comme étant une entité exerçant durablement une activité économique sans regard à sa forme juridique ou son mode de financement. ${ }^{151}$ Une entreprise ainsi définie peut alors regrouper, dans le cadre du droit européen des pratiques anticoncurrentielles, plusieurs personnes morales si celles-ci agissent sur le marché intérieur ou sur un marché national ou régional comme une seule entité économique. ${ }^{152}$ Par contre, le destinataire d'une décision de la Commission Européenne (concernant par exemple

${ }_{147}$ Hunout, L'entreprise et le droit du travail - Une comparaison franco-allemande, 1993.

148 Notamment Despax (n. 2), p. 265 et s et 377 et s.; A. Lyon-Caen/G. Lyon-Caen (n. 136), p. 599 et s.; Friedel, A propos de la notion d'entreprise, in: Aspects actuels du droit commercial français - Études dédiées à René Roblot, 1984, p. 97 et s.

149 Vischer/Rapp, Zur Neugestaltung des schweizerischen Aktienrechts, 1968, p. 145 et s.; Druey (n. 137), n. 100 et s.

150 Blaise (n. 3), n. 349 ("... l'entreprise n'est que l'instrument de l'infraction, mais n'en est pas la responsable ....); Blanc (n. 133), p. 416 et s. (entreprise enjeu de l'application de la norme).

151 Se référer pour cette jurisprudence constante à p. ex. CJCE du 23 avril 1991 aff. C-41/90, Rec. 1991, p. I-1979 (Höfner et Elser).

152 CJCE du 6 mars 1974 aff. 6 et 7/73, Rec. 1974, p. 223 n. 39 (Commercial Solvents/Kommission); CJCE du 25 octobre 1983 aff. 107/82, Rec. 1983, p. 3151 n. 50 (AEG/Kommission); CJCE du 12 juillet 1984 aff. 170/83, Rec. 1984, p. 2999, n. 11 et s. (Hydrotherm/ Compact). 
une amende) ne peut être qu’une personne ayant la personnalité juridique ou au moins la capacité juridique.

Nous pouvons constater la même approche en matière du contrôle exercé sur les acteurs des marchés financiers. Par exemple, selon la directive 2006/48/ $\mathrm{CE}$, les États membres et les autorités de surveillance compétentes peuvent renoncer à l'application des exigences de fonds propres sur une base individuelle et appliquer ces exigences sur une base consolidée en évaluant un groupe de sociétés comme étant une seule entité économique lorsqu'ils le jugent approprié. ${ }^{153}$ Par contre, les destinataires des décisions des autorités de surveillance compétentes restent les différents membres du groupe. ${ }^{154}$

\section{L'entreprise en droit pénal}

En droit pénal, les droits germaniques ont longtemps refusé d’accepter une responsabilité pénale des personnes morales et notamment de l'entreprise. ${ }^{155} \mathrm{Bien}$ que le législateur allemand reste fidèle à cette position, selon laquelle il n'y a pas de peine sans faute (nulla poena sine culpa), ${ }^{156}$ le législateur suisse a introduit en 2003 une disposition prévoyant une responsabilité pénale des entreprises. ${ }^{157}$ Si un crime ou un délit est commis au sein d'une entreprise dans l'exercice d'activités commerciales conformes à ses buts et si ce crime ou ce délit ne peut

153 Se référer au considérant 13 et aux art. 68 et s. de la directive 2006/48/CE.

154 Se référer aux art. 124 al. 1,136 et s. de la directive 2006/48/CE.

155 Se référer pour cette doctrine classique notamment à $v$. Savigny, System des heutigen römischen Rechts, t. II, 1840, p. 312: «Das Criminalrecht hat zu thun mit dem natürlichen Menschen, als einem denkenden, wollenden, fühlenden Wesen. Die juristische Person aber ist kein solches, sondern nur ein Vermögen habendes Wesen, liegt also ganz außer dem Bereich des Criminalrechts.».

156 Se référer encore au Bundesministerium der Justiz (éd.), Abschlußbericht der Kommission zur Reform des strafrechtlichen Sanktionensystems, 2000, p. 199 et s.; bien que les art. 6 et s. de la directive 2008/99/CE du Parlement Européen et du Conseil du 19 novembre 2008 relative à la protection de l'environnement par le droit pénal (JOUE $\mathrm{n}^{\circ} \mathrm{L} 328 \mathrm{du}$ 6/12/2008, p. 28 et s.) demandent aux États membres d'introduire une responsabilité pénale des personnes morales, le législateur allemand juge suffsant les dispositions des $\$ \$ 30$ et 130 OWiG prévoyant une responsabilité administrative des exploitants d'une entreprise (se référer aux motifs du projet de loi du 6 avril 2011, BTDrucks. 17/5391, p. 15).

157 Art. $100^{\text {quater }}$ ancien Code pénale suisse (aujourd'hui art. $102 \mathrm{CP}$ ); voir Bertossa, Unternehmensstrafrecht - Strafprozess und Sanktionen, 2003; Macaluso, La responsabilité pénale de l'entreprise - Principes et commentaire des art. $100^{q^{\text {quater }}}$ et $100^{\text {quinquies }} \mathrm{CP}, 2004$; pour la situation juridique avant 2003 Heine, Die strafrechtliche Verantwortlichkeit von Unternehmen - Von individuellem Fehlverhalten zu kollektiven Fehlentwicklungen, insbesondere bei Großrisiken, 1995. être imputé à aucune personne physique déterminée en raison du manque d'organisation de l'entreprise, ce crime ou ce délit sera imputé à l'entreprise qui sera punie d'une amende de cinq millions de francs suisses au plus. Dans le cadre de certaines infractions particulières (p. ex. blanchiment d'argent ou corruption), lentreprise peut être punie indépendamment de la responsabilité des personnes physiques, si on peut lui reprocher de ne pas avoir pris toutes les mesures d'organisation raisonnables et nécessaires pour empêcher une telle infraction. Le juge fixe l'amende entre autres d'après la capacité économique de l'entreprise. Bien que ce soit l'entreprise en tant qu'entité économique qui est prise en considération pour déterminer si l'activité en question rentre dans son champ d'activité et si la non-identification d'un responsable physique relève de son manque d'organisation, ce sera l'exploitant en tant que sujet de droit qui sera le destinataire de la punition. La notion d'exploitant se trouve à l'al. 4 de l'art. $102 \mathrm{CP}$, qui est curieusement intitulé «entreprises au sens du présent titre». Les personnes morales de droit privé et de droit public à l'exception des corporations territoriales, les sociétés et les entreprises à titre individuel sont des entreprises au sens de l’al. 4 de l'art. 102 CP.

\section{Conclusion}

Pour terminer cette esquisse sur l'entreprise et les droits européens, il convient de mentionner deux tendances allant en faveur d'un droit de lentreprise:

La première tendance peut être intitulée du droit commercial au droit de l'entreprise. L'entrepreneur prend de plus en plus le relais du commerçant. L'exemple du législateur autrichien a déjà été mentionné. Il a transformé son droit commercial - repris en 1938 du droit allemand - en un droit de l'entreprise (UGB) et a ouvert son champ d'application aux exploitants agricoles, forestiers et de profession libérale. Même le législateur allemand, traditionnellement attaché à la notion de commerçant, ${ }^{158}$ procède de plus en plus, sous l'influence du droit international ${ }^{159}$ et européen, ${ }^{160}$ à une distinction bipartite entre l'entrepreneur ou le professionnel et le consommateur. ${ }^{161}$ Au vu de cette distinction, le commerçant n'est plus qu'une sous-forme de l'entrepreneur ou du professionnel.

158 Siems, Kaufmannsbegriff und Rechtsfortbildung, $2^{\mathrm{e}}$ éd., 2003.

159 Se référer à l'exemple des art. 1 et 2 let. a de la Convention des Nations Unies sur les contrats de vente internationale de marchandises du 11 avril 1980 (CVIM).

160 Se référer à l'exemple de la directive 93/13/CEE concernant les clauses abusives dans les contrats conclus avec les consommateurs.

161 Se référer à titre d'exemple aux $\$ \$ 13,14 \mathrm{BGB}$ avec $\$ 310 \mathrm{BGB}$ (conditions générales) et $\$ 491$ al. 1 BGB (crédit à la consommation).

Peter Jung 
La deuxième tendance peut être intitulée du droit des sociétés au droit de l'entreprise. ${ }^{162}$ Tandis qu'en droit des sociétés l'application des normes dépend en règle générale de la forme juridique de la société, le droit de lentreprise ne fait pas de distinction entre les différentes formes juridiques de l'exploitant mais s'applique en fonction de critères quantitatifs concernant l'entreprise tel que le chiffre d'affaire, le résultat du bilan et le nombre de salariés. La loi allemande relative à la publicité des entreprises ${ }^{163}$ représente un bon exemple. Selon cette loi de 1969, les exploitants de certaines entreprises importantes doivent rendre public leurs comptes annuelles indépendamment de leurs formes juridiques. En droit suisse, les sociétés remplissant certains critères de dimension sont soumises depuis 2008 à une vérification de leurs comptes annuels par un organe de révision selon les art. 727 et s. du Code des obligations.

Le droit de l’entreprise va ainsi être amené à jouer un rôle au côté du droit commercial et du droit des sociétés.

\section{Première Partie:}

Identité nationale et convergence des droits

162 Rappr. le titre d'un compte rendu collectif de Geßler, Vom Gesellschafts- zum Unternehmensrecht, ZHR 143 (1979), 427 et s.

163 Gesetz über die Rechnungslegung von bestimmten Unternehmen und Konzernen (PublG) du 5 août 1969, BGBl. 1969 I, p. 1189 et s. 